\title{
Link Scheduling Algorithm to Mitigate Worst-Case Interference Problems for LTE-A
}

\author{
Shivanandaswamy V. Mahapurush ${ }^{* 1}$, Prathima Mahapurush ${ }^{2}$, Priyatam Kumar ${ }^{3}$ \\ ${ }^{1}$ Assistant Professor, Department of Electronics and Communication Engineering, Smt Kamala \& Sri \\ Venkappa M. Agadi College of Engineering \& Technology, Lakshmeshwar, Gadag, Karnataka, India \\ ${ }^{2}$ Assistant Professor, Department of Information Science and Engineering, Smt Kamala \& Sri Venkappa M. \\ Agadi College of Engineering \& Technology, Lakshmeshwar, Gadag, Karnataka, India \\ ${ }^{3}$ Professor, School of Electronics and Communication Engineering, KLE Technological University, \\ Vidyanagar, Hubli, Karnataka, India \\ *Corresponding Author: shivu1201@ gmail.com
}

\begin{abstract}
As the number of apps increases, the traffic of multimedia services in wireless networks grows exponentially. Because of the proportional fair algorithm's shortcomings in terms of achieving Quality of Service (QoS) requirements such as delay and packet loss, it is not extensively employed in modern wireless communication. This study provides a scheduling method based on the QoS utility function, with a parameter added to improve the priority mechanism in order to overcome such flaws. Our scheduling algorithm can match the QoS criteria of real-time traffic, according to simulation results, and the overall system transmission performance has increased significantly.
\end{abstract}

Key words: Quality of service, Scheduling algorithm, Utility function, Proportional fair

\section{INTRODUCTION}

To meet the demands of fast-processing applications operating on a wide range of internet-connected wireless devices such as smart phones, laptops, and palmtops, among others. In LTE Release 10, the Third Generation Partnership Project (3GPP) proposed carrier aggregation technology. ITU has standardized carrier aggregation as a fourth-generation technological component for achieving the target data rates of $1 \mathrm{Gbps}$ in the downlink [1] and $500 \mathrm{Mbps}$ in the uplink [2]. Wider bandwidths of up to $100 \mathrm{MHz}$ spectrum are required to achieve such large data rates [2]. The 3GPP LTE systems with transmission bandwidths of $1.4 \mathrm{MHz}$, $4 \mathrm{MHz}, 5 \mathrm{MHz}$, and $20 \mathrm{MHz}$ served as the foundation for the planned $100 \mathrm{MHz}$ IMT-Advanced mobile systems, also known as LTE Advanced. Multiple LTE carrier components (CCs) with varying bandwidths and situated in the same or distinct frequency bands can be aggregated in CA to obtain a bandwidth of up to 100 $\mathrm{MHz}$ for supplying user equipment with downlink and uplink transmission speeds of up to 1 Gbps and 500 Mbps, respectively. By merging the unused or surplus secondary carrier components of one cell with the primary carrier component of a geographically different cell (both of which are supplied by distinct base stations) $[2$,$] the unused or surplus secondary carrier components$ of one cell can be efficiently utilized. This allows for more efficient use of the wireless world's limited radio resources (spectrum).

\section{LITERATURE SURVEY}

A practical scheduler should be capable of increasing overall system capacity while maintaining some level of user fairness $[4,5,8,11]$. Several packet scheduling algorithms have recently been proposed in order to deliver faster throughput while ensuring fairness to all users. The scheduling mechanism in [6], for example, uses Assignment Model to achieve this balance by effectively mapping UEs to RBs at each TTI. The suggested algorithm in [6] modifies the Best-Channel Quality Indicator (Best-CQI) method, which is characterized by high data rates at the cell level but poor fairness, in order to achieve fairness in resource distribution while keeping system capacity utilization as high as possible. The authors of [9] present a multi-user scheduling system that operates on the edge of the feasible multi-user rate region while ensuring a desirable long-term average fairness. [1] implemented another scheduling algorithm based on the utility function in order to enhance the performance of the LTE system, specifically the throughput and fairness performance. By splitting the packet scheduler into a time domain and a frequency domain and using separate algorithms in both domains, as described in [2], throughput fairness among users may be successfully controlled. [4] Describes a Generalized Proportional Fair (GPF) scheduling methodology and its application to OFDMA frequency scheduling. When compared to a system without 
frequency scheduling, this improves system throughput while also improving fairness in terms of allotted resources and obtained data rates per user. [4] Proposes an adaptive proportional fair scheduling method for LTE systems, in which the author attempts to create an algorithm that promotes UE fairness while limiting overall system throughput degradation by altering scheduling priority according to individual user's channel condition.

\section{PROPOSED METHODOLOGY}

LTE was introduced as an upgrade of the Universal Mobile Telecommunication Systems (UMTS) in order to provide cellular network users with high data rates in the uplink and downlink directions, low latency, and efficient spectrum usage [3]. The correct scheduling algorithm that satisfies the environment's characteristics and the users' requirements requests could be used to maximize spectrum utilization.

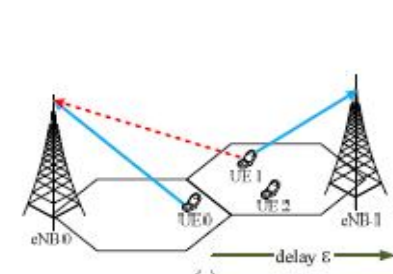

(a)

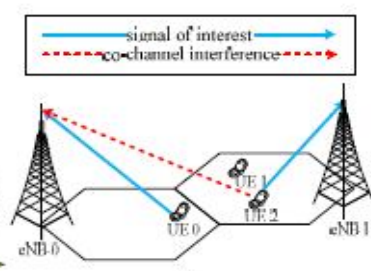

(b)
Figure 1:

System model for SU-MIMO UL transmission

The coordinator anticipates the next epoch's interference state based on the SINR, current status, and number of neighbors at each epoch. The coordinator will broadcast a negotiation message to the network if the next status of a WBAN is ShortIF. The coordinators exchange the TDMA schedule in the super-frame for sharing the transmission channel in the interference avoidance process after receiving signals from the neighbors.

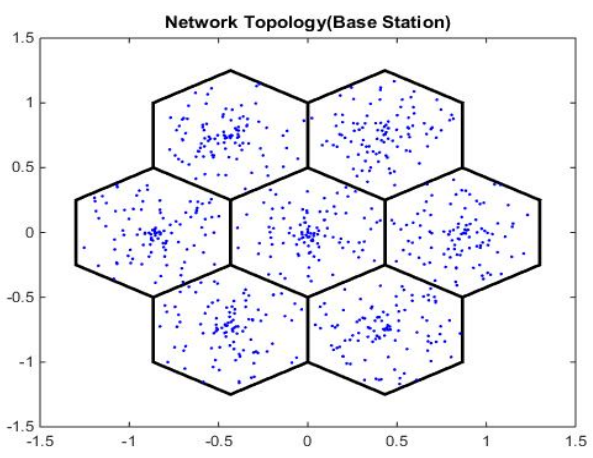

Figure 2: Network Topology
It's worth noting that the FFR or SFR schemes' prospective benefits aren't always achieved [5, 8]. On the one hand, ICIC can reduce interference for celledge users, increasing the bit rate of cell-edge users. In a unity FRF system, on the other hand, the lower SINR caused by ICI can be compensated by assigning extra bandwidth to these users. Even without ICIC, a satisfactory performance can be attained by compensating for the reduced SINR with greater bandwidth, that is, resource blocks, to suitable users using an intelligent scheduler. In this scenario, the extra complexity of inter-cell interactions may not justify the advantages of ICIC [9]. However, as [10] points out, the benefit of bandwidth compensation is not completely realized if a limit is set on the peak rate that can be employed for a specific bearer. ICIC has been proven to be most useful for low-tomoderate traffic levels. CQI feedback frequency is a tradeoff between temporal diversity gain and effective interference reduction achieved by a frequency-selective scheduler. More frequent CQI feedback allows for better temporal adaptation to channel variance, resulting in increased spectral efficiency. The frequency of CQI feedback, on the other hand, should be determined by the short-term interference caused by surrounding cells' instantaneous scheduling decisions. The effectiveness of CQI feedbacks, particularly those from cell-edge users, is considerably diminished because this interference is weakly associated with that in succeeding sub-frames. As a result, a less frequent scheduling decision might be beneficial in terms of interference minimization. CQI feedback filtering, according to [12], should be used to average out the temporal changes of the ICI, resulting in a more stable CQI value for cell-edge users.

Because RP offers superior system performance and short-term fairness than other algorithms [4], we design an improved PF scheduling algorithm based on the riding peaks (RP) technique under successive RBs allocation restriction [4].

UE k's improved riding peaks (IRP) PF factor fk (i,t) at sub-band $I$ and time slot $t$ is defined as:

$$
f_{k}(i, t)=\lambda_{t}(i, t) * \beta_{t}(i, t)
$$

Where we introduce a multiplicative factor $\beta_{\mathrm{k}}(\mathrm{i}, \mathrm{t})$ in the traditional PF metric formula. This factor is defined as:

$$
\beta_{t}(i, t)=\left\{\begin{array}{cc}
1 \quad k=u(i, t-1) \\
\theta & \text { ot } \square \text { ers }
\end{array}, \theta \in\right.
$$

$(0,1)$ 
Where is a constant factor that can be set at eNB. Each eNB saves the last (time slot $t-1$ ) scheduling results of each sub-band to reduce UL ICI variance and increase AMC accuracy. The following is a representation of the scheduling history:

$$
H(t)=\{u(i, t-1) \mid i \in \Lambda, u(i, t-1) \in \Omega\}(3)
$$

The collection of sub-bands is shown as $u(i, t-1)$, where $\mathrm{u}(\mathrm{i}, \mathrm{t}-1)$ is the UE scheduled on sub-band I during time slot $t$. When eNBs conduct the resource allocation on sub-band I at the present moment (time slot $\mathrm{t}$ ), the scheduling controllers conduct the following:

- Calculate the traditional PF metric $\lambda_{\mathrm{t}}(\mathrm{i}, \mathrm{t})$ for UE $\mathrm{k}$ on sub-band $\mathrm{i}$ at time slot $\mathrm{t}$, as shown in (7).

- Calculate the Improved PF metric $f_{k}(i, t)$ as shown in (13). Compare UE $\mathrm{k}$ with the history scheduling information $\mathrm{u}(\mathrm{i}, \mathrm{t}-1)$. If $\mathrm{k}$ is not equal $\mathrm{u}(\mathrm{i}, \mathrm{t}-1)$, then we give UE $\mathrm{k}$ a lower priority with the value of $\theta$. Otherwise, our improved PF metric is identical to the traditional one, as shown in (10).

$$
f_{t}(i, t)-\left\{\begin{array}{cc}
\lambda_{k}(i, t) * \theta \quad k \neq u(i, t-1) \\
\lambda_{k}(i, k) & \text { others }
\end{array}\right.
$$

We give lesser priority to some UEs who have not been scheduled on the target sub-band in the past two steps (time slot t-1). To put it another way, some UEs have the same PF metric value. The user who was scheduled on the previous TTI will be scheduled by the eNB. The ICI variance caused by the frequent changes of scheduled UEs with identical PF factors in a few adjacent TTIs can be reduced using this strategy. The entire algorithm is shown below:

\section{METHODOLOGY:}

\section{ALGORITHM:}

Improved Proportional Fair Scheduling Algorithm for LTE

Initialization:

The set of unallocated sub-bands: $S=\Lambda$;

The set of sub-bands allocated to UE $k: M_{k}=\varphi$;

$H(t)=\varphi ;$

Assume that each UE occupies the whole bandwidth and compute the initial improved $P F$ factor $f_{i, k}(t)$ for each UE $k$ according to (6);

Let $V$ be the sorted list of all the metric value $f_{i, k}(t)$

in decreasing order; $m=1$.

While $S \neq \varphi$ do

Pick sub-band $i$ with $m$-th largest metric value $f_{i, k} \in V$ ;

if ( $i$ is adjacent to $\left.M_{k}\right)$ or $\left(M_{k}=\varphi\right)$ then

Assign sub-band i to UE $k$;

$\mathrm{S}=\mathrm{S}-\{\mathrm{i}\}$

$\mathrm{Mk}=\mathrm{Mk}+[\mathrm{i}\}$

$H(t+1)=H(t+1)+\{u(i, t)\} u(i, t)=k\}$

$V=V-\left\{f_{k}(i, t)\{k \in \Omega\}\right.$

$m=1$

else

$m=m+1$.

end if

end while 


\section{RESULTS}

The highest throughput is achieved by our suggested Improved Proportional Fair Scheduling Algorithm, which is followed by RP TDA and standard RP. At CAUT and CEUT, gains of roughly 29 percent and 35 percent can be reached with an equivalent of 0.8 .

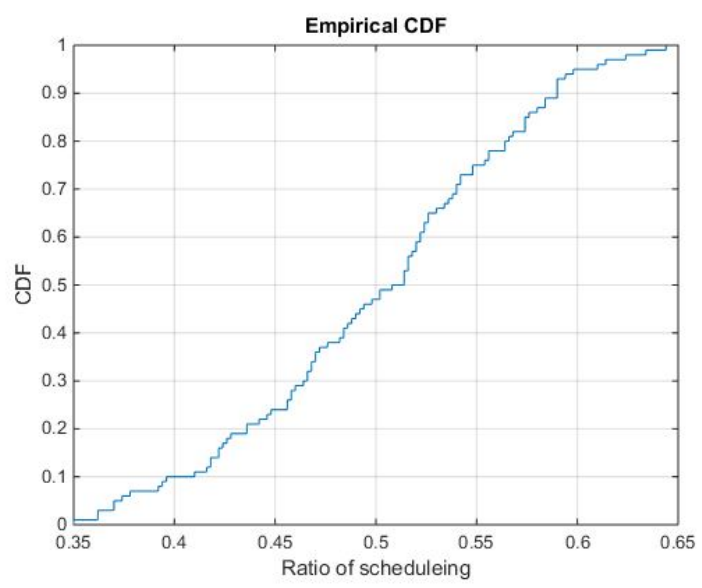

Figure 3: Ratio of scheduling vs CDF

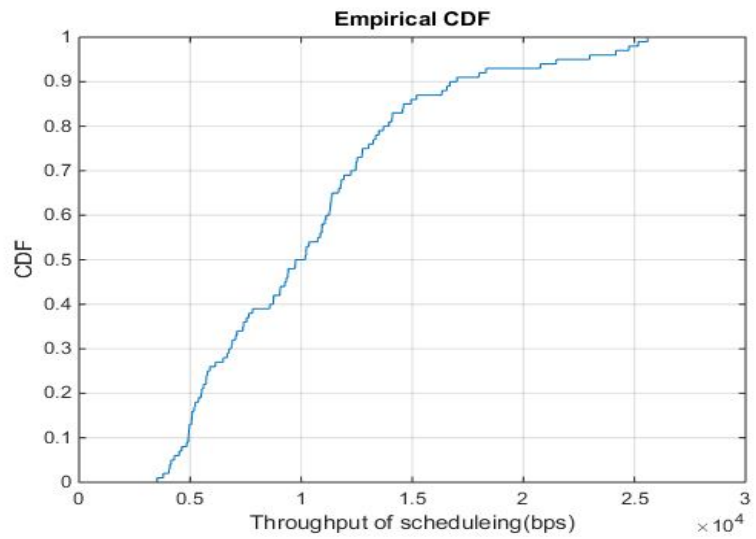

Figure 4: System throughput CDF

The gain of the CAUT and the chance of initial transmission success grow steadily as the value of falls, whereas the gain of the CEUT declines. These patterns appear to be in line with our assumptions. The lower the value of $\theta$, the lower the priority of the UEs that were not scheduled at the previous TTI. Because of the frequency selective scheduling, the
CEUT gain will be reduced. Furthermore, the increased chances of first transmission success and CAUT suggest a better fit between MCS level selection and instantaneous channel condition. Because the IRP method performs better on CAUT and CEUT, we need to examine short-term fairness (less than $100 \mathrm{msec}$ ).

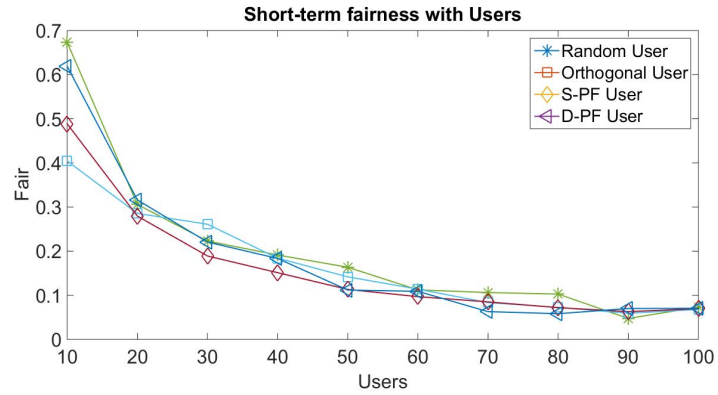

Figure 5: Variation of Fairness of scheduler with users

When the time period is less than $20 \mathrm{msec}$, our suggested approach with an equivalent to 0.8 has greater short-term fairness than RP and RP TDA. Otherwise, it performs similarly. When the time interval is smaller than $40 \mathrm{msec}$, the IRP algorithm is commendable with a value of less than 0.8 . When the time period is greater than $40 \mathrm{msec}$, however, it has a lower short-term fairness. Furthermore, RP TDA performs worse than RP in terms of short-term fairness, despite the former's superior performance at CAUT and CEUT.

\section{REFERENCES}

[1] 3GPP TS 36.213, "Evolved Universal Terrestrial Radio Access (E-UTRA), Physical layer procedures (Release 10)," TS 36.213 v10.10.0, 2013. [2] A. Ubisse and N. Ventura, "Modeling a link level simulator for long term evolution uplink," in The Southern Africa Telecommunication Networks and Applications Conference (SATNAC '11), East London, South Africa, September 2011. 
[3] 3GPP TS 36.104, "Evolved Universal

Terrestrial Radio Access (E-UTRA), Base Station (BS) radio transmission and reception (Release 10),” TS 26.104 v10.11.0, 2013.

[4] A. Sadek and S. Shellhammer, "SUI Path-Loss

Model for Coexistence Study," IEEE 802.1908/0010r0, IEEE P802.19 Wireless Coexistence, 2008.

[5] 3GPP TS 36.101, "Evolved Universal Terrestrial Radio Access (E-UTRA), User Equipment (UE) radio transmission and reception (Release 10),” TS 36.101 v10.11.0, 2013.

[6] K. Konstantinou, S. Kang, and C. Tzaras, "A measurement based model for mobile-to-mobile UMTS links," in Proceedings of the IEEE 65th Vehicular Technology Conference (VTC '07), pp. 529-533, Dublin, Ireland, April 2007.

[7] T. Nihtila and V. Haikola, HSDPA Performance with Dual Stream MIMO in a Combined MacroFemto Cell Network, IEEE VTC 2010.

[8] H. R. Karimi et al., Evolution Towards Dynamic Spectrum Sharing in Mobile Communications, IEEE PIMRC 2006.
[9] G. Boudreau et al., Interference Coordination and Cancellation for 4G Networks, IEEE Commun. Mag., 2009.

[10] A. Khandekar et al., LTE-Advanced: Heterogeneous Networks, European Wireless Conf. 2010.

[11] O.Simeone, E.Erkip, S.Shamai, Robust Transmission and Interference Management For Femto-cells with Unreliable Network Access, IEEE JSAC, Dec. 2010.

[12] S. Annapureddy et al., http://www.ieeectw.org/2010/mon/Gorokhov.pdf, 2010 IEEE Commun.Theory Wksp., Cancun, Mexico, 2010. 